no point of $B$. Let $f(z)$ be analytic and bounded in $G . A$ necessary and sufficient condition for the existence of polynomials $\left\{p_{n}(z)\right\}$ which converge to $f(z)$ in $G$ so that (1) holds is that there exist a function analytic and bounded in $\Gamma$ and equal to $f(z)$ in $G$.

The proof of this theorem is much the same as for Theorem A taken together with the remark of $\$ 5$ in the earlier paper and is therefore omitted.

The conclusion of Theorem D simply means of course that $f(z)$ shall be analytically extensible throughout $\Gamma$.

Union College

\title{
A GENERALIZED PARSEVAL'S RELATION
}

BY E. S. QUADE

A function $\phi(x)$ which is non-negative, convex, and satisfies the conditions $\phi(0)=0$ and $(\phi(x) / x) \rightarrow \infty$ as $x \rightarrow \infty$ will be called a Young's function. Given such a function $\phi(x)$, a second function, $\psi(x)$, with the same properties can be found such that Young's inequality, $a b \leqq \phi(a)+\psi(b)$, holds for every $a, b \geqq 0$. The functions $\phi(x)$ and $\psi(x)$ are then said to be complementary in the sense of Young. $\dagger$

If $x(t)$ is such that $\int_{a}^{b} \phi(|x|) d t$ exists, $x(t)$ is said to belong to the space $L_{\phi}(a, b)$. This space is not necessarily linear. $f$ For this reason we denote by $L_{\phi}^{*}(a, b)$ the class of all functions $x(t)$, $a \leqq t \leqq b$, such that the product $x(t) y(t)$ is integrable for every $y(t) \epsilon L_{\psi}(a, b)$. If we put

$$
\|x\|_{\phi}=\sup _{y}\left|\int_{a}^{b} x(t) y(t) d t\right|
$$

for all $y(t)$ with

$$
\rho_{y} \equiv \int_{a}^{b} \psi(|y|) d t \leqq 1,
$$

then $L_{\phi}^{*}$ is a linear metric, and complete space. $\S$ A function

$\dagger$ W. H. Young, Proceedings Royal Society, (A), vol. 87 (1912), pp. 225229.

$\ddagger \mathrm{W}$. Orlicz, Über eine gewisse Klasse von Räumen vom Typus B, Bulletin, Académie Polonaise, (A), Cracovie (1932).

§ A. Zygmund, Trigonometrical Series, 1935, pp. 95-100. 
$x(t) \epsilon L_{\phi}^{*}$ can be approximated by its Féjer polynomials; $\dagger$ that is, given $\epsilon>0$, there exists an $N_{0}(\epsilon)$ such that

$$
\left\|x-\sigma_{n}\right\|_{\phi} \leqq \epsilon, \quad n \geqq N_{0}(\epsilon),
$$

where $\sigma_{n}=\sigma_{n}(t ; x)$ is the $n$th $(C, 1)$ mean of the Fourier series of $x(t)$. Since $\sigma_{n}(t ; x)$ is continuous, there exists a step function $\omega_{n}(t)$ which is a linear combination of simple step functions such that $\left|\sigma_{n}(t ; x)-\omega_{n}(t)\right| \leqq \epsilon$ for $a \leqq t \leqq b$. Then

$$
\begin{aligned}
\left\|x-\omega_{n}\right\|_{\phi} & \leqq\left\|x-\sigma_{n}\right\|_{\phi}+\left\|\sigma_{n}-\omega_{n}\right\|_{\phi} \\
& \leqq \epsilon+\sup _{y}\left|\int_{a}^{b}\left[\sigma_{n}(t)-\omega_{n}(t)\right] y(t) d t\right| \\
& \leqq \epsilon+\epsilon\left(\sup _{y} \int_{a}^{b}|y(t)| d t\right) \\
& \leqq \epsilon+\epsilon[\phi(1)(b-a)+1]=M \epsilon,
\end{aligned}
$$

where $M$ is independent of $x(t)$. This implies that the set of simple step functions is a fundamental set in the space $L_{\phi}{ }^{*}$.

In the following we take $(0,1)$ as the fundamental interval and $\left\{K_{n}(s, t)\right\}$ to be a sequence of measurable kernels defined on the unit square.

THEOREM 1. Necessary and sufficient conditions that

$$
\int_{0}^{1} y(s) d s \int_{0}^{1} K_{n}(s, t) x(t) d t \rightarrow \int_{0}^{1} x(s) y(s) d s
$$

for every pair $x(t) \epsilon L_{\psi}^{*}, y(s) \epsilon L_{\phi}^{*}$ are as follows.

(1) If $\delta=(a, b)$ and $\pi=(\alpha, \beta)$ are any subintervals of $(0,1)$, then

$$
\begin{gathered}
\int_{a}^{b} d s \int_{\alpha}^{\beta} K_{n}(s, t) d t \rightarrow \operatorname{meas}(\delta \cdot \pi) ; \\
\left\|\int_{0}^{1} K_{n}(s, t) x(t) d t\right\|_{\psi} M\left\|_{x}\right\|_{\psi},
\end{gathered}
$$

where $M$ is independent of $n$. $\ddagger$

$\dagger$ A. Zygmund, loc. cit., p. 107 (14).

$\ddagger$ For analogous results in some more special cases we refer to J. C. Burhill, Strong and weak convergence of functions of general type, Proceedings London Mathematical Society, (2), vol. 28 (1928), pp. 493-500; Z. W. Birnbaum and W. Orlicz, Über die Verallgemeinerung des Begriffes der zueinander konjugierten Potenzen, Studia Mathematica, vol. 3 (1931), pp. 1-67; W. Orlicz, loc. cit. 
Since the set of simple step functions is a fundamental set, the necessity of (1) is obvious. In order that the integral

$$
\int_{0}^{1} y(s) d s \int_{0}^{1} K_{n}(s, t) x(t) d t
$$

exist for arbitrary $y(s) \epsilon L_{\phi}^{*}$,

$$
U_{n}(x) \equiv \int_{0}^{1} K_{n}(s, t) x(t) d t
$$

must belong to $L_{\psi}{ }^{*}$ for arbitrary $x(t) \epsilon L_{\psi}{ }^{*}$ and $n$. We wish to show that $U_{n}(x)$ is a linear operation on $L_{\psi}{ }^{*}$ to $L_{\psi}{ }^{*}$. We need the following lemmas.

LemmA A. $\lim _{n \rightarrow \infty}\left\|x_{n}\right\|_{\psi}=0$ implies lim $\operatorname{asymp}_{n \rightarrow \infty} x_{n}(t)=0$.

Set $y_{n}(t)=a \operatorname{sign} x_{n}(t)$, where $a>0, \psi(a) \leqq 1$. Then

$$
\begin{aligned}
\left\|x_{n}\right\|_{\psi} & =\sup _{y}\left|\int_{0}^{1} y(t) x_{n}(t) d t\right| \geqq\left|\int_{0}^{1} y_{n}(t) x_{n}(t) d t\right| \\
& =a \int_{0}^{1}\left|x_{n}(t)\right| d t \rightarrow 0 .
\end{aligned}
$$

But

$$
\lim _{n \rightarrow \infty} \int_{0}^{1}\left|x_{n}(t)\right| d t=0 \text { implies } \lim \underset{n \rightarrow \infty}{\operatorname{asymp}} x_{n}(t)=0 .
$$

LemmA B. $\lim _{n \rightarrow \infty}\left\|x_{n}\right\|_{\psi}=0$ implies the existence in $L_{\psi}{ }^{*}$ of a subsequence $\left\{x_{n_{i}}(t)\right\}$ and an $x(t)$ such that $\left|x_{n_{i}}(t)\right| \leqq|x(t)|$ for every $i=1,2,3, \cdots$, and almost all $t$.

Let $\left\|x_{n}(t)\right\|_{\psi} \rightarrow 0$. Without loss of generality we may take each $x_{n}(t) \geqq 0$. Then there exists a subsequence $\left\{x_{n_{i}}(t)\right\} \subset\left\{x_{n}(t)\right\}$ such that $\sum_{i=1}^{\infty}\left\|x_{n_{i}}\right\|_{\psi} \leqq M$, where $M$ is a constant. This means that $\sum_{i=1}^{\infty} x_{n_{i}}(t)$ converges in the space $L_{\psi}{ }^{*}$ to a function $x(t) \in L_{\psi}{ }^{*}$. Then

$$
\left|x_{n_{i}}(t)\right|=x_{n_{i}}(t) \leqq x(t)
$$

Lemma C. $\lim \operatorname{asymp}_{n \rightarrow \infty} x_{n}(t)=x(t)$ implies $\underline{\lim }_{n \rightarrow \infty}\left\|x_{n}\right\|_{\psi} \geqq\|x\|_{\psi}$. 
Suppose the contrary. Then there exists a subsequence $\left\{x_{n_{i}}(t)\right\}$ such that $\lim _{i \rightarrow \infty}\left\|x_{n_{i}}\right\|_{\psi}<\|x\|_{\psi}$. This means that

$$
\sup _{y}\left|\int_{0}^{1} x_{n_{i}} y d t\right| \leqq\|x\|_{\psi} \text { which implies } \int_{0}^{1}\left|x_{n_{i}} y\right| d t \leqq\|x\|_{\psi}
$$

for all $i$ sufficiently large. Since $\lim$ asymp ${ }_{i \rightarrow \infty} x_{n_{i}}(t)=x(t)$, there exists a subsequence $x_{n_{i}}(t) \rightarrow x(t)$ almost everywhere. Hence $\left|x_{n_{i}}(t) y(t)\right| \rightarrow|x(t) y(t)|$ almost everywhere for each fixed $y(t)$. By the Fatou Lemma we have

$$
\varliminf_{i \rightarrow \infty} \int_{0}^{1}\left|x_{n_{i}}^{\prime} y\right| d t \geqq \int_{0}^{1}|x y| d t \geqq\left|\int_{0}^{1} x y d t\right| .
$$

Since this must hold for every $y(t)$, we have

$$
\lim _{i \rightarrow \infty}\left\|x_{n_{i}}^{\prime}\right\|_{\psi} \geqq \sup _{y} \lim _{i \rightarrow \infty} \int_{0}^{1}\left|x_{n_{i}}^{\prime} y\right| d t \geqq\|x\|_{\psi}
$$

which means that $\lim _{i \rightarrow \infty}\left\|x_{n_{i}}\right\|_{\psi} \geqq\|x\|_{\psi}$, a contradiction.

Consequently, by a general theorem of Banach, $\dagger U_{n}(x)$ is a linear operation. Hence $\left\|U_{n}(x)\right\|_{\psi} \leqq M_{n}\|x\|_{\psi}$. But the sequence

$$
\left\{\left|\int_{0}^{1} y(s) d t \int_{0}^{1} K_{n}(s, t) x(t) d t\right|\right\}
$$

is bounded (for each fixed $x(t) \epsilon L_{\psi}^{*}$ ) for every $y(s) \epsilon L_{\phi}^{*}$. This implies $\ddagger\left\|U_{n}(x)\right\|_{\psi} \leqq M(x)$ independently of $n$. Then, by the BanachSteinhaus theorem,

$$
\left\|U_{n}(x)\right\|_{\psi}=\left\|\int_{0}^{1} K_{n}(s, t) x(t) d t\right\|_{\psi} \leqq M\|x\|_{\psi},
$$

where $M$ is independent of $n$.

To prove the sufficiency we set

$$
\int_{\alpha}^{\beta} K_{n}(s, t) d t=\int_{0}^{1} K_{n}(s, t) w_{\alpha \beta}(t) d t=w_{n}(s),
$$

† S. Banach, Théorie des Opérations Linéaires, 1932, p. 87.

‡ A. Zygmund, loc. cit., p. 99. 
where $w_{\alpha \beta}(t)$ is the characteristic function of the interval $(\alpha, \beta)$. From (1) we have

$$
\int_{a}^{b} w_{n}(s) d s \rightarrow \int_{a}^{b} w_{\alpha \beta}(s) d s .
$$

Let

$$
f_{n}(y)=\int_{0}^{1} y(s) d s \int_{\alpha}^{\beta} K_{n}(s, t) d t=\int_{0}^{1} y(s) w_{n}(s) d s .
$$

Then if $\lambda \equiv M\left\|w_{\alpha \beta}\right\|_{\psi}$ and $\rho_{w_{n} / \lambda}^{\prime}=\max \left(1, \rho_{w_{n} / \lambda}^{\prime}\right)$, since

$$
\rho_{w_{n} / \lambda} \equiv \int_{0}^{1} \psi\left(\frac{1}{\lambda}\left|w_{n}(t)\right|\right) d t \leqq 1,
$$

we have $\dagger$

$$
\left|f_{n}(y)\right| \leqq \lambda\|y\|_{\phi} \rho_{w_{n} / \lambda}^{\prime} \leqq M\left\|w_{\alpha \beta}\right\|_{\psi}\|y\|_{\phi},
$$

where the right-hand side of the inequality is independent of $n$. Hence from a general theorem on linear functionals, $\ddagger$ we have

$$
\int_{0}^{1} y(s) w_{n}(s) d s \rightarrow \int_{0}^{1} y(s) w_{\alpha \beta}(s) d s
$$

for arbitrary $y(s) \epsilon L_{\phi}^{*}$. Now set

$$
g_{n}(x)=\int_{0}^{1} y(s) d s \int_{0}^{1} K_{n}(s, t) x(t) d t .
$$

This gives a sequence of linear functionals defined on $L_{\psi}{ }^{*}$. For every $w_{\alpha \beta}(s)$,

$$
g_{n}\left(w_{\alpha \beta}\right) \rightarrow g\left(w_{\alpha \beta}\right) \equiv \int_{0}^{1} y(s) w_{\alpha \beta}(s) d s .
$$

Moreover, since§

$$
\left|g_{n}(x)\right| \leqq \rho_{y}^{\prime}\left\|\int_{0}^{1} K_{n}(s, t) x(t) d t\right\|_{\psi} \leqq \rho_{y}^{\prime} M\|x\|_{\psi},
$$

$\dagger$ A. Zygmund, loc. cit., p. 97 .

$\ddagger$ S. Banach, loc. cit., p. 123, Theorem 2.

$\S$ A. Zygmund, loc. cit., p. 97. 
we see that the modulus of the functional $g_{n}(x)$ is $\leqq M_{\rho_{y}^{\prime}}$ independently of $n$, and we may again apply the theorem on functionals used above to obtain the desired result.

As a corollary of Theorem 1, we have the following theorem.

TheOREM 2. Necessary and sufficient conditions that

$$
\int_{0}^{1} y(s) d s \int_{0}^{1} K_{n}(s, t) x(t) d t \rightarrow \int_{0}^{1} y(s) x(s) d s
$$

for every pair $y(s) \epsilon L_{p}, x(s) \epsilon L_{p^{\prime}},(1<p<\infty)$, are:

$$
\begin{gathered}
\int_{a}^{b} d s \int_{\alpha}^{\beta} K_{n}(s, t) d t \rightarrow \text { meas }(\delta \cdot \pi) ; \\
\left(\int_{0}^{1}\left|\int_{0}^{1} K_{n}(s, t) x(t) d t\right|^{p^{\prime}} d s\right)^{1 / p^{\prime}} \leqq M\|x\|_{p^{\prime}} .
\end{gathered}
$$

[For the case $x(s) \epsilon L, y(s) \epsilon M \equiv L_{\infty}$, the theorem holds provided we replace in (1) the interval $(a, b)$ by the arbitrary measurable set $E$. The proof for this case is essentially the same as that of Theorem 1.]

TheOREM 3. Necessary and sufficient conditions $\dagger$ that

$$
\int_{0}^{1} y(s) d s \int_{0}^{1} K_{n}(s, t) x(t) d t \rightarrow \int_{0}^{1} y(s) x(s) d s
$$

for every pair $y(s) \epsilon L, x(t) \epsilon M$ are:

$$
\begin{aligned}
& \int_{a}^{b} d s \int_{E} K_{n}(s, t) d t \rightarrow \text { meas } \delta \cdot E ; \\
& \underset{s}{\text { ess sup }} \int_{0}^{1}\left|K_{n}(s, t)\right| d t \leqq M .
\end{aligned}
$$

Except for the necessity of (2) the proof is exactly that of Theorem 1. As in Theorem 1 we have

$$
\underset{s}{\operatorname{ess} \sup }\left|\int_{0}^{1} K_{n}(s, t) x(t) d t\right| \leqq M^{\prime}\|x\|_{M} .
$$

$\dagger$ J. D. Tamarkin, Zentralblatt, vol. 10 (1935), pp. 349-350, in review of paper by I. Natanson, Bulletin, Société de Physique et Mathématique, Kazan, vol. 3 (1934). 
Letting $x(t)=e(t)$ be the characteristic function for the set $E$, we have

$$
\underset{s}{\operatorname{ess} \sup }\left|\int_{0}^{1} K_{n}(s, t) d t\right| \leqq M^{\prime} .
$$

This implies $†$ that (2) holds.

If, in Theorem 2, condition (2) were replaced by either

$$
\begin{aligned}
\left(\int_{0}^{1}\left(\int_{0}^{1}\left|K_{n}(s, t)\right|^{p} d t\right)^{p^{\prime} / p} d s\right)^{1 / p^{\prime}} & \leqq M, \\
& (n=1,2, \cdots) .
\end{aligned}
$$

or

$$
\begin{aligned}
\left(\int_{0}^{1}\left(\int_{0}^{1}\left|K_{n}(s, t)\right|^{p^{\prime}} d s\right)^{p / p^{\prime}} d t\right)^{1 / p} \leqq M, & \\
& (n=1,2, \cdots),
\end{aligned}
$$

the conditions of Theorem 2 would be sufficient but no longer necessary. For example, the Dirichlet kernel, $D_{n}(s, t)=D_{n}(t,-s)$, (where the fundamental interval is $(0,2 \pi)$ and the functions are assumed periodic) does not satisfy either of these conditions but does satisfy those of Theorem 2 . We note that for $p \geqq 2$, $\left(2^{\prime \prime}\right)$ implies $\left(2^{\prime}\right)$ and for $1<p \leqq 2,\left(2^{\prime}\right)$ implies $\left(2^{\prime \prime}\right)$.

THEOREM 4. Sufficient conditions that

$$
\int_{0}^{1} y(s) d s \int_{0}^{1} K_{n}(s, t) x(t) d t \rightarrow \int_{0}^{1} y(s) x(s) d s,
$$

where $x(s) \epsilon L_{\psi}^{*}, y(s) \epsilon L_{\phi}^{*}$ are:

$$
\begin{aligned}
& \int_{a}^{b} d s \int_{\alpha}^{\beta} K_{n}(s, t) d t \rightarrow \text { meas }(\delta \cdot \pi) ; \\
& \operatorname{ess} \sup _{s} \int_{0}^{1}\left|K_{n}(s, t)\right| d t \leqq M, \quad(n=1,2, \cdots) ; \\
& \operatorname{ess} \sup \int_{0}^{1}\left|K_{n}(s, t)\right| d s \leqq M, \quad(n=1,2, \cdots) .
\end{aligned}
$$

† Saks and Tamarkin, Annals of Mathematics, vol. 34 (1933), p. 600. Theorem 2. 
We set $u_{n}(s)=\int_{0}^{1} K_{n}(s, t) x(t) d t$ and consider

$$
\frac{u_{n}(s)}{M}=\int_{0}^{1}\left(\frac{K_{n}(s, t)}{M}\right) x(t) d t .
$$

Since

$$
\operatorname{ess} \sup _{s} \int_{0}^{1}\left|K_{n}(s, t)\right| d t \leqq 1
$$

and $\psi(0)=0$, we have by Jensen's inequality

Consequently

$$
\begin{aligned}
\psi\left(\frac{\left|u_{n}(s)\right|}{M\|x\|_{\psi}}\right) & =\psi\left(\left|\int_{0}^{1} \frac{K_{n}(s, t)}{M} \cdot \frac{x(t)}{\|x\|_{\psi}} d t\right|\right) \\
& \leqq \psi\left(\int_{0}^{1}\left|\frac{K_{n}(s, t)}{M}\right| \cdot\left|\frac{x(t)}{\|x\|_{\psi}}\right| d t\right) \\
& \leqq \frac{1}{M} \int_{0}^{1} \psi\left(\frac{|x(t)|}{\|x\|_{\psi}}\right)\left|K_{n}(s, t)\right| d t .
\end{aligned}
$$

$$
\begin{aligned}
\int_{0}^{1} \psi\left(\frac{\left|u_{n}(s)\right|}{M\|x\|_{\psi}}\right) d s & \leqq \frac{1}{M} \int_{0}^{1} d s \int_{0}^{1} \psi\left(\frac{|x(t)|}{\|x\|_{\psi}}\right)\left|K_{n}(s, t)\right| d t \\
& \leqq \frac{1}{M} \int_{0}^{1} \psi\left(\frac{|x(t)|}{\|x\|_{\psi}}\right) d t \int_{0}^{1}\left|K_{n}(s, t)\right| d s \\
& \leqq \int_{0}^{1} \psi\left(\frac{|x(t)|}{\|x\|_{\psi}}\right) d t \leqq 1
\end{aligned}
$$

We have thus shown that $u_{n}(s)=\int_{0}^{1} K_{n}(s, t) x(t) d t$ takes $x(t) \epsilon L_{\psi}^{*}$ into $u_{n}(s) \epsilon L_{\psi}^{*}$ for arbitrary $n$, and hence condition (2) of Theorem 1 follows as in the proof of the necessity in that theorem.

BROWN UNIVERSITY 